\title{
Drug-coated balloon treatment of late scaffold thrombosis and proliferated neointima and atherosclerotic plaque
}

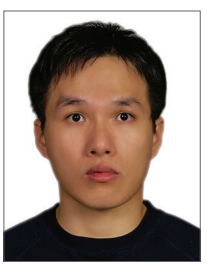

Wei-Chieh Lee, MD; Hsiu-Yu Fang, MD; Chiung-Jen Wu*, MD

Division of Cardiology, Department of Internal Medicine, Kaohsiung Chang Gung Memorial Hospital, Chang

Gung University College of Medicine, Kaohsiung, Taiwan
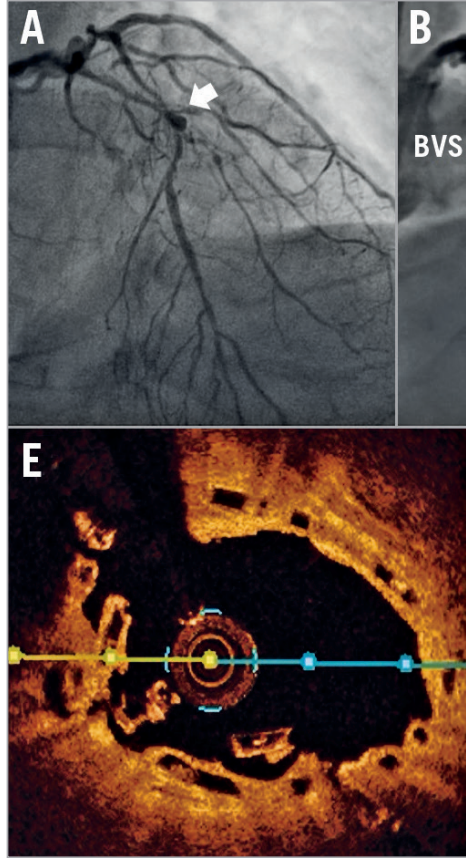
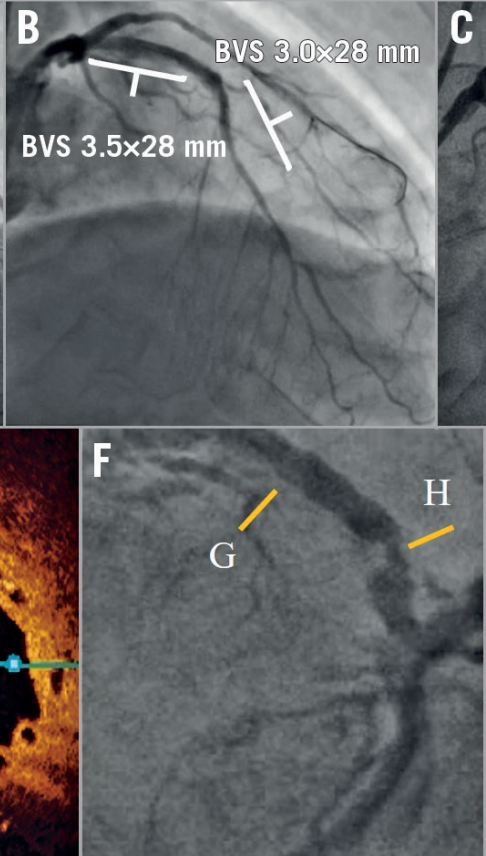
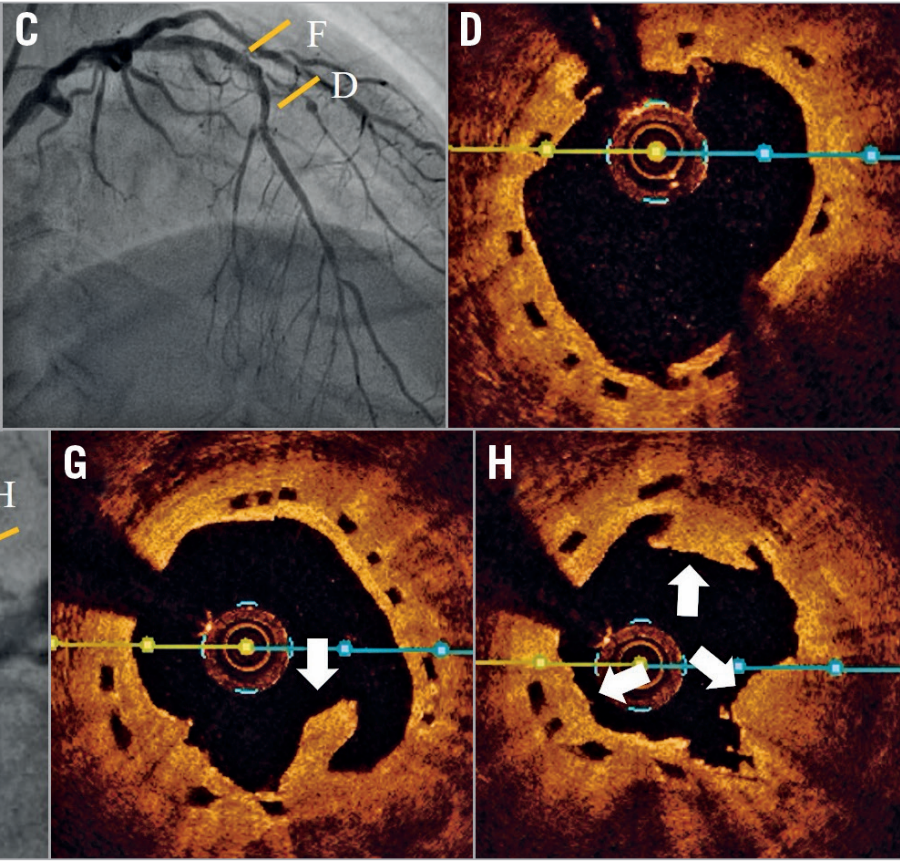

H
A 68-year-old man experienced unstable angina, and had type 2 diabetes mellitus and hyperlipidaemia. The left anterior descending artery (LAD) showed long stenotic lesions with calcification, and one aneurysm at the mid segment (Panel A). After intravascular ultrasound for pre-sizing, predilatation was performed with a high-pressure balloon. One $3.0 \times 28 \mathrm{~mm}$ bioresorbable vascular scaffold (BVS; Abbott Vascular, Santa Clara, CA, USA) was deployed at the mid LAD at $16 \mathrm{~atm}$, and another $3.5 \times 28 \mathrm{~mm}$ BVS was deployed at the proximal LAD at $16 \mathrm{~atm}$ (Panel B). After adequate post-dilatation, optical coherence tomography (OCT) showed a good apposition. One year later, the patient experienced typical angina symptoms; follow-up angiography and OCT imaging showed no stenosis at the mid LAD (Panel C, Panel D). OCT imaging did however show poor absorption at the overlapping site (Panel E), one severe in-stent restenosis (ISR) with haziness at the proximal LAD (Panel F), and proliferated neointima with atherosclerotic plaque rupture and some thrombi in the previous BVS of the proximal LAD (Panel G, Panel H). One $3.5 \times 30 \mathrm{~mm}$ SeQuent Please ${ }^{\circledR}$ drug-coated balloon (DCB; B. Braun Melsungen AG, Melsungen, Germany) was inserted and inflated. Dual antiplatelet therapy was prolonged for two years and the patient did not present with any angina symptoms. A thallium perfusion scan showed a negative ischaemic finding. The BVS has been proved to be feasible for complex and calcified lesions. However, BVS absorption and endothelial growth may differ in different individuals. The use of a DCB for BVS thrombosis with proliferated neointima and atherosclerotic plaque has not been reported previously. We have reported here a case using DCB treatment for inscaffold neointima proliferation.

\section{Conflict of interest statement}

The authors have no conflicts of interest to declare.

*Corresponding author: Division of Cardiology, Department of Internal Medicine, Kaohsiung Chang Gung Memorial Hospital, Niao Sung District, Kaohsiung City, Taiwan.E-mail: leeweichieh@yahoo.com.tw 